\title{
The Structure of Dendrimers with Charged Terminal Groups: Monte Carlo Simulations
}

\author{
M. MAJTYKA ${ }^{a}$ AND J. KŁOS ${ }^{b, c, *}$ \\ ${ }^{a}$ Max-Planck-Institute for Polymer Research \\ Postfach 3148, 55021 Mainz, Germany \\ ${ }^{b}$ Faculty of Physics, A. Mickiewicz University \\ Umultowska 85, 61-614 Poznań, Poland
}

${ }^{c}$ Leibniz Institute for Polymer Research Dresden e.V., 01069 Dresden, Germany

(Received July 28, 2006)

\begin{abstract}
Taking into account the full Coulomb potential and the excluded volume interactions, properties of dendrimers with generations $g=5,6$ with charged terminal groups and counterions in an athermal solvent are examined by lattice Monte Carlo simulations. The study treats counterions explicitly and focuses on the local structure of the systems inspected by pair correlation functions $g_{a b}$ that provide information on distributions of monomers, terminal groups and ions in space at various temperatures $T^{*}$. Special emphasis is placed on counterions and their role they play in causing conformational changes of the molecules. The simulations show that counterions penetrate the interior of the dendrimers, and there is a major increase in their concentration there as $T^{*}$ decreases. Some of them condense onto the terminal groups and a reduction in the mean effective charge $\langle Q\rangle$ of the dendrimers appears. Within the range of temperatures where the condensation (as a function of $T^{*}$ ) is sharp the molecules weakly swell up when compared to their size at the other temperatures. This kind of behaviour is also reflected by the distributions of monomers and terminal groups.
\end{abstract}

PACS numbers: 82.20.Wt, 82.35.Lr, 82.35.Rs

\section{Introduction}

There has been an increasing interest in examining starburst dendrimers $[1,2]$ with charged terminal groups. This is mostly because these molecules provide a rich potential for applications in industry, biomedicine, pharmacy, and materials engineering. Some examples of their use include lithographic materials, nanoscale catalysts, drug delivery systems, rheology modifiers, bioadhesives, and magnetic

*e-mail: klosj@o2.pl 
resonance imaging (MRI) contrast agents [3]. Though experimental studies [4-9], theory and simulations [10-14] have already brought about some insight into the way charged dendrimers react to various conditions, the research on them is far from being completed. For instance, the number of simulation papers is still very limited and some predictions reported in them contrast with experimental results. For instance, it is still rather unclear if the dendrimer size is sensitive to the charge density or ionic strength of the solvent. On one hand, simulations indicate such a dependence, whereas small-angle neutron scattering does not [5].

Furthermore, since the Debye-Hückel potential has been applied in most calculations [12-14], the possibility of ion condensation onto the molecule (a phenomenon of great importance in the case of linear polyelectrolytes [15-34]) has been excluded a priori. It turns out, however, that this issue must be handled with great care. The question of the role of counterions treated explicitly has only recently been taken up by means of molecular dynamics and lattice Monte Carlo simulations $[35,36]$. In spite of different models and computational methods used, the overall pictures reported correspond well. With increasing strength of electrostatic interactions counterion condensation occurs and makes a tremendous impact on the structure of dendrimers that change their size nonmonotonically by subsequent swelling and shrinking.

In this paper we take an interest not only in shape and size of the dendrimers but also in local properties of the whole systems inspected by pair correlation functions (PCF) $g_{a b}$. We report on lattice Monte Carlo simulations carried out for end-charged dendrimers with higher generations $g=5,6$ accompanied by neutralizing counterions and for varying strength of the electrostatic interaction referred to as the reduced temperature $T^{*}$. This allows us to inspect systematically distributions of the free ions in the vicinity of the dendrimers in relation to their conformations.

The remaining part of the paper is organized as follows. First, the simulation method and the quantities of interest are outlined. Then, the results of our simulations are presented and discussed. Finally, some conclusions and remarks are given.

\section{Model and numerical procedure}

The systems contain a single dendrimer and counterions on a face centered cubic lattice with periodic boundary conditions in all three dimensions. A dendrimer is a tree-like collection of beads connected by rigid, nonbreakable bonds of length $a=u \sqrt{2}$ (where $u$ is the length unit) so as to form the macromolecular skeleton (for a 2D schematic representation see Fig. 1). Before simulation runs, dendrimers with generations $g=5,6$, spacer length $P=1$, and branching functionality $m=3$ are formed by a growth process starting from the initiator. Thus, the molecules have $N_{5}=254, N_{6}=510$ monomers and $N_{5 \mathrm{t}}=128, N_{6 \mathrm{t}}=256$ terminal groups, respectively. Furthermore, each end-bead carries a positive charge 


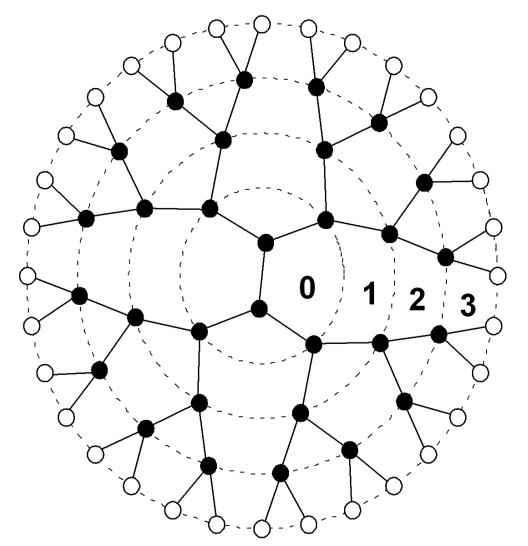

Fig. 1. Schematic 2D picture of dendrimers (supplied by T. Pakula).

of valence $z=1$, whereas $N_{g \mathrm{t}}(g=5,6)$ free beads (counterions) negative charges $z=-1$. The solvent is implicitly included by its permittivity $\varepsilon$.

The electrostatic interaction between the charges is the total Coulomb energy

$$
E_{\mathrm{c}}=\delta E=\frac{1}{2} \delta \sum_{i, j=1, i \neq j}^{2 N_{g \mathrm{t}}} \frac{z_{i} z_{j}}{r_{i j}}
$$

where $r_{i j}$ is the distance between the $i$-th and $j$-th ions with valences $z_{i}, z_{j}$, and $\delta=e^{2} / \varepsilon u$ is the energy unit with $e$ denoting the electric charge. The long-range nature of the Coulomb interactions is treated by the Ewald summation method with the minimum image convention for the real-space term, $\kappa=5 / L, k_{\max }=5$ for the sum in the reciprocal space and for a conducting external medium [37]. In the paper, we calculate thermodynamic averages at the reduced temperature $T^{*}$ defined as $T^{*}=k_{\mathrm{B}} T / \delta$, where $T$ is the absolute temperature and $k_{\mathrm{B}}$ is the Boltzmann constant. Furthermore, the excluded volume condition is taken into account by preventing a lattice site from being occupied by more than one element. Thus, the dendrimers are in an athermal solvent.

In the Monte Carlo scheme configurations are tossed using the generalized version of the cooperative motion algorithm (CMA) [35]. The tossing scheme used can be outlined in the following way. (1) A bead (monomer, counterion) and one of its neighbouring lattice sites are selected at random. (2) If the latter is empty an elementary CMA move is performed. In case the selected bead is a counterion it is translated to the empty site. This way another empty site (the abandoned one) is generated which is the end of the trial reconfiguration for single, free beads. In case the selected bead is a monomer, it is moved to the empty site as well. Furthermore, depending on the local situation encountered, in order that no bonds are broken and that the sequence of beads on the dendrimer is preserved, a number of monomers that follow the selected one are also shifted 
collectively one after another by subsequent translating by one lattice site until an empty site is finally generated. This is the end of the trial reconfiguration for the monomers. (3) If the lattice site selected in (1) is occupied, the whole of step (1) is repeated. A new configuration is accepted or rejected according to a probability of the Metropolis type [38]

$$
p=\min \left[1, \exp \left(-\frac{\Delta E}{T^{*}}\right)\right],
$$

with $\Delta E=E_{\text {new }}-E_{\text {old }}\left(E_{\text {new }}\right.$ and $E_{\text {old }}$ are energies after and before a random reconfiguration, $E=E_{\mathrm{c}} / \delta$ ). In the simulations, we have computed the mean-square radius of gyration

$$
\left\langle R_{\mathrm{g}}^{2}\right\rangle=\left\langle\frac{1}{N} \sum_{i=1}\left(r_{i}-r_{\mathrm{c} . \mathrm{m} .}\right)^{2}\right\rangle,
$$

the mean internal energy $\langle E\rangle=\left\langle E_{\mathrm{c}}\right\rangle / \delta$, and the mean effective charge per end-bead

$$
\langle Q\rangle=1-\frac{\left\langle N_{\mathrm{c}}\right\rangle}{N_{g \mathrm{t}}}
$$

In (3), $r_{i}$ and $r_{\text {c.m. }}$ are the position vectors of the $i$-th monomer and of the centre of mass (c.m.) of the dendrimer, respectively. In (4), $\left\langle N_{\mathrm{c}}\right\rangle$ is the mean number of condensed counterions (a counterion is considered condensed when it occupies a nearest-neighbour site of at least one end-bead), and the bracket \langle\rangle stands for thermodynamic averaging. The PCF $g_{a b}$ are defined as

$$
g_{\alpha \beta}(r)=\frac{\left\langle N_{\alpha \beta}(r+u)\right\rangle}{N_{\alpha} N_{\beta}^{\mathrm{id}}(r+u)},
$$

where the numerator denotes the mean number of pairs of elements of type $\alpha$ and $\beta$ at the distance from each other between $r$ and $r+u, N_{\alpha}$ is the total number of elements $\alpha$, and $N_{\beta}^{\mathrm{id}}(r+u)$ is the mean number of elements $\beta$ in an ideal gas in the same interval. In the paper the PCFs include the c.m.-monomer PCF $g_{\text {c.m.m }}$, c.m.--end-bead PCF $g_{\text {c.m. e+ }}$, monomer-monomer PCF $g_{\mathrm{m} \mathrm{m}}$, end-bead-counterion PCF $g_{\mathrm{e}+\mathrm{c}-}$, and the counterion-counterion $\mathrm{PCF} g_{\mathrm{c}-\mathrm{c}-}$, respectively.

The simulations have been performed on a $3 \mathrm{D}$ fcc lattice of the size $L^{3}=$ $80 \times 80 \times 80 u^{3}$ for systems of single dendrimers in a wide range of temperatures $T^{*}$. Mostly, configurations obtained for some $T^{*}$ were initial ones for further calculations at lower $T^{*}$.

\section{Results}

We shall start by reporting on the phenomenon of counterion condensation onto the terminal groups and the configuration changes it causes for the dendrimers. In Fig. 2a, we plot the mean effective charge per end-bead $\langle Q\rangle$ versus $T^{*}$ for both generations $g=5,6$. The monotonic decrease in $\langle Q\rangle$ with $T^{*}$ clearly indicates that the effect of condensation does occur, indeed. Roughly, for $0.1<T^{*}<10$ (the dotted line), the reduction in $\langle Q\rangle$ becomes sharper and 
$0<\langle Q\rangle\langle 1$, whereas at the other temperatures $\langle Q\rangle$ is practically one and zero. Obviously, $\langle Q\rangle=0$ corresponds to the high-temperature limit without condensed ions, whereas $\langle Q\rangle=1$ to perfect condensation at lower temperatures. The tendency of counterions to form a "cloud" around the dendrimer and to settle on it is also well seen in Fig. 3. At given temperature some of them are still distributed in space, whereas others are already condensed on the molecule. Furthermore, according to Fig. 2b, the ion condensation is accompanied by a monotonic decrease in $\langle E\rangle$. The states with perfect condensation are ones with the lowest energies. Below, we argue that this kind of observation is likely to offer an explanation for the low-temperature configurational changes of the dendrimers.



Fig. 2. The effective mean charge per end-bead $\langle Q\rangle$ (a), the mean electrostatic energy $\langle E\rangle$ (b), and the mean-square radius of gyration $\left\langle R_{\mathrm{g}}^{2}\right\rangle$ (in units of $u^{2}$ ) (c) versus $T^{*}$. (a)-(c) are for $g=5,6$, the dotted lines correspond to temperatures at which the dendrimer is swollen.

Next, we take a look at the size of the molecules as a function of $T^{*}$. This is shown in Fig. 2c by the mean-square radius of gyration $\left\langle R_{\mathrm{g}}^{2}\right\rangle$. For $0.1<T^{*}<10$, $\left\langle R_{\mathrm{g}}^{2}\right\rangle$ approaches a broad maximum for both generations. Thus, the molecules weakly swell up (the change in size is smaller than $10 \%$ ) for the same temperatures where the condensation is sharp as a function of $T^{*}$ and partial $(0<\langle Q\rangle<1)$. Therefore, it seems reasonable to conjecture that in this temperature regime the electrostatic repulsion between like-charged end-beads is dominant and the largest separations between them, i.e., extended branches, are statistically favoured. In 


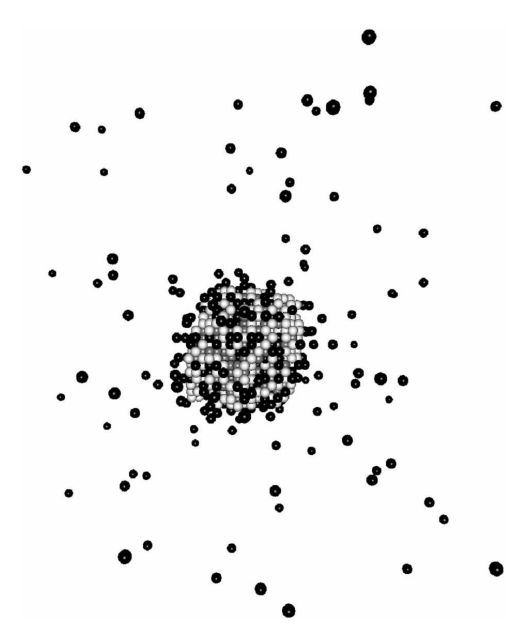

Fig. 3. The configuration snapshot for $g=6$ and for $T^{*}=1$. The black, grey, and white spheres represent counterions, end-beads, and neutral monomers, respectively.

the limit of high $T^{*}$, the interactions are effectively weak, and all the beads behave like neutral, self-avoiding particles. Due to perfect condensation at the lowest temperatures, almost all counterions settle on the molecule. Since such states have the lowest energies, grouping of similar charges in space is statistically unfavourable. Instead, charges with mixed valences (positive and negative) are more likely to gather close to each other which, in turn, promotes local attraction between various parts of the molecule and reduction in its size. Thus, at least with respect to higher strength of the electrostatic interaction, counterions cannot be omitted from being taken into consideration.

In what follows we turn to distributions of monomers and ions as seen by the PCFs. The distribution of the former as a function of the distance $r$ from the c.m. of the dendrimer for $g=5,6$ and $T^{*}$ ranging from high to low are shown in Fig. 4a. For all of the inspected temperatures, there is a high concentration of monomers close to the origin which corresponds to the dense core of the molecules. Then, for increasing $r, g_{\text {c.m.m }}$ drops down sharply to a local minimum and subsequently increases to a broad, local maximum $[12,14,36,39-44]$ that is flatter for the higher generation $g=6$. The dotted lines in the figure show that due to swelling, the concentration of monomers tends to shift down inside the dendrimer, whereas for temperatures at which the molecules are more shrunken the monomers are packed in a denser way. Finally, on the periphery of the molecules $g_{\text {c.m.m }}$ decreases to zero. The concentrations of terminal groups relative to the c.m. of the dendrimers are shown in Fig. 4b. The plots clearly show that to some extent the terminal groups enter the interior of the dendrimers. Actually, $g_{\text {c.m. e+ grows from finite }}$ value at the core to a broad maximum in the interior and falls down to zero for larger $r$. Moreover, due to the effect of swelling for $0.1<T^{*}<10$ (the dotted lines), $g_{\text {c.m. e+ }}$ is smaller inside the molecules and larger on the periphery than in 


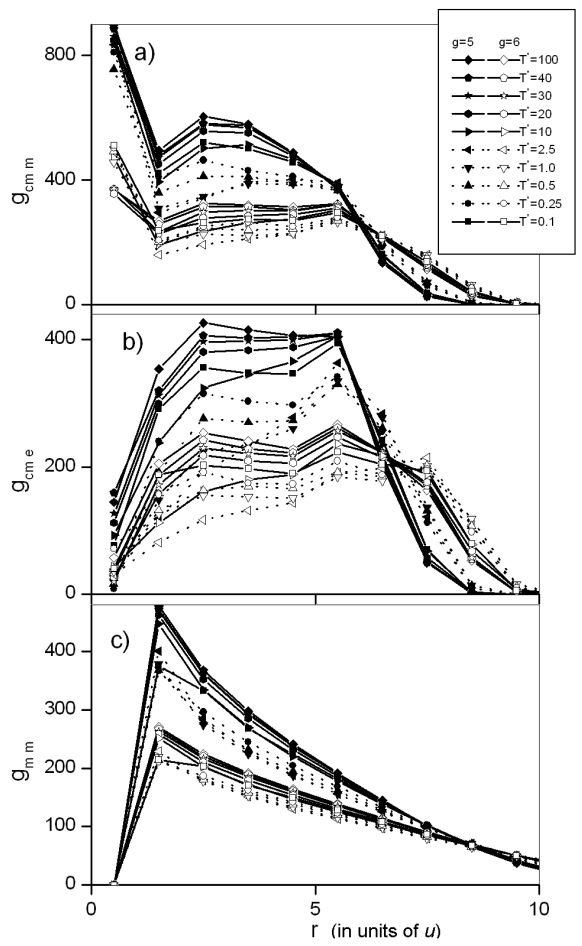

Fig. 4. The c.m.--monomer PCF $g_{\text {c.m.m }}$ (a), the c.m.-end-bead PCF $g_{\text {c.m.e }}$ (b), and the monomer-monomer PCF $g_{\mathrm{m} \mathrm{m}}$ (c) at various $T^{*}$. (a)-(c) are for $g=5,6$, the dotted lines correspond to temperatures at which the dendrimer is swollen.

the shrunken states. Finally, Fig. 4c shows the monomer-monomer PCFs $g_{\mathrm{mm}}$ (in this case $r$ denotes the distance between pairs of monomers). In the first shell they have sharp peaks that decrease for given $r$ due to looser packing of the monomers in the swelling range. Thus, $g_{\text {c.m. e+ }}, g_{\text {c.m.m }}$ and $g_{\mathrm{mm}}$ correspond well with the size and shape changes of the dendrimer.

On the basis of Fig. 5a presenting the c.m.-counterion PCF $g_{\text {c.m.c- }}$ for $g=5$, we conclude that counterions not only condense onto the terminal groups but also penetrate the whole interior of the molecule. Actually, there is a major increase in their concentration inside the dendrimer as $T^{*}$ decreases, whereas close to the c.m. and on the periphery $g_{\text {c.m. c- }}$ decreases sharply. It is also interesting to see that near the c.m. $g_{\text {c.m. c- }}$ is still finite, which means that a number of counterions are permanently present there. Furthermore, in accordance with the earlier studies [36], Fig. 5b shows that, due to strong condensation of counterions onto the end-beads at the lowest $T^{*}, g_{\mathrm{c} . \mathrm{m} . \mathrm{c}-}$ and $g_{\mathrm{c} . \mathrm{m} . \mathrm{e}+}$ practically overlap. Then, as $T^{*}$ increases, condensation becomes partial and the plots distinguishable. At this point it is also worth stressing that some experimental techniques such as electron paramagnetic resonance (EPR), transmission electron microscopy (TEM), small- 


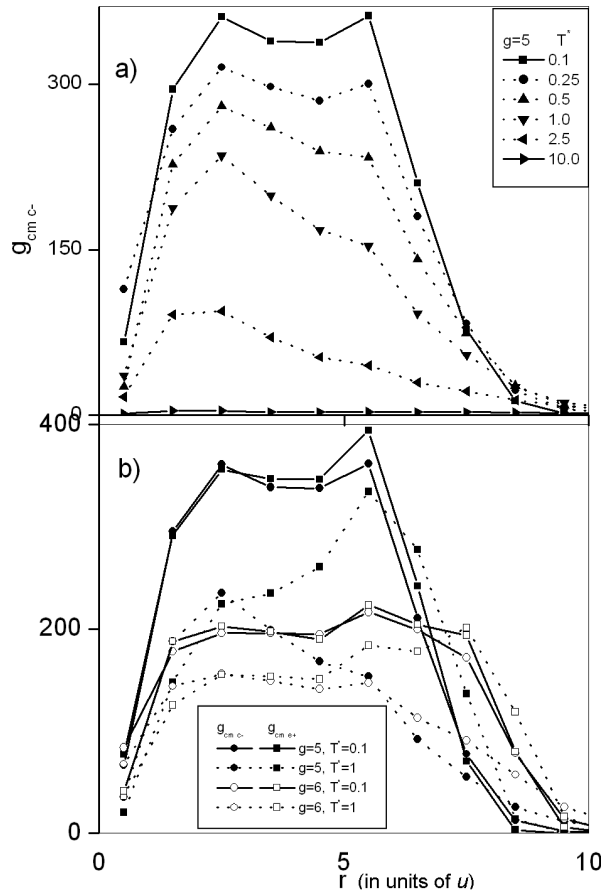

Fig. 5. The c.m.-counterion PCF $g_{\text {c.m.c- }}$ at various $T^{*}$ for $g=5$ (a), the c.m.-end-bead PCF $g_{\text {c.m. e+ }}$ and the c.m.-counterion PCF $g_{\text {c.m.c- }}$ for $g=5,6$ and for $T^{*}=$ $0.1,1$ (b).

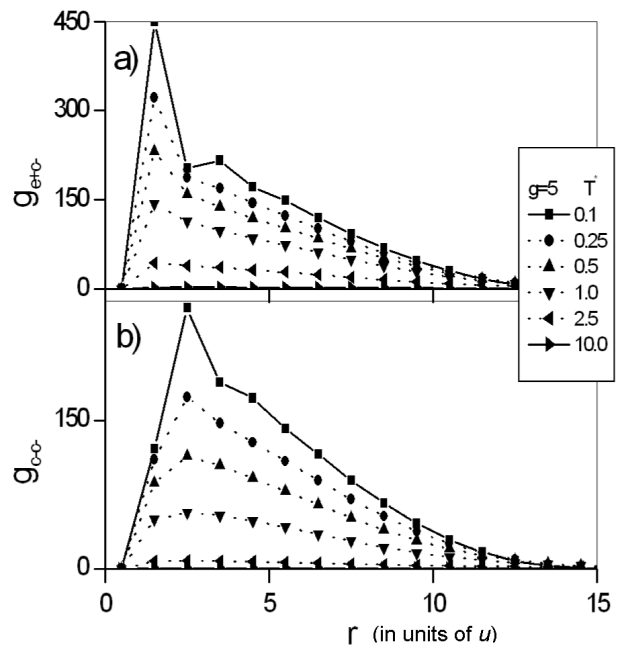

Fig. 6. The end-bead-counterion PCF $g_{\mathrm{e}+\mathrm{c}-}$ (a), and the counterion-counterion PCF $g_{\mathrm{c}-\mathrm{c}-}(\mathrm{b})$, at various $T^{*}$. (a), (b) are for $g=5$, the dotted lines correspond to temperatures at which the dendrimer is swollen. 
-angle neutron scattering (SANS), and small-angle X-ray scattering (SAXS) have already enabled an inspection of the above described phenomena. In particular, similar migration of ions to the molecule's interior has already been detected for poly(amidoamine) (PAMAM) dendrimers and metal ions $(\mathrm{Cu}, \mathrm{Pd}, \mathrm{Au})$ [45-49].

Finally, the distributions of counterions in the vicinity of the end-beads and around each other are shown in Figs. 6a,b, respectively. The end-bead-counterion PCF $g_{\mathrm{e}+\mathrm{c}-}$ clearly confirms that counterions tend to gather near the terminal groups as $T^{*}$ is lowered. Actually, the curves possess sharp maxima for very small interparticle distances that are higher for decreasing $T^{*}$. Similar behaviour reveals the counterion-counterion PCF $g_{\mathrm{e}-\mathrm{c}-}$ as well. Due to condensation the particles are brought close to each other and peaks in the PCF occur.

\section{Summary}

In this paper, we have carried out Monte Carlo simulations of single dendrimers with charged, monovalent terminal groups, and counterions for varying strength of the electrostatic interaction. Among others, the calculations have provided information on the distributions of counterions, monomers, and terminal groups in space. Counterions penetrate the interior of the molecule, and as the temperature decreases, their concentration there becomes considerably greater than in the bulk. Furthermore, they condense onto the end-beads reducing the effective charge of the dendrimer and causing its conformational changes of swelling and shrinking. As already emphasized, all our observations are in agreement with the very recent studies based on off-lattice simulations. This suggests that the importance of counterions and their role in the described phenomena seem fundamental and independent of the models used.

The effects discussed in this paper are due to wide variations in strength of the electrostatic interaction $T^{*}$ itself. However, this kind of approach might be difficult to be realized experimentally, especially in the limit of low $T^{*}$. Therefore, it seems reasonable to inspect other possibilities of causing conformational changes of dendrimers. In particular, we expect to observe important results for systems with explicit counterions and added salt ions. For example, of special interest here is the effect of salt concentration and salt valence on the properties of end-charged dendrimers. These issues will be addressed in another paper.

\section{Acknowledgments}

This work was supported by by the State Committee for Scientific Research (grant MEIN 24/6 PR UE/2006/7). The authors wish to acknowledge the Poznań Supercomputing and Networking Center where the calculations were performed. The authors are very grateful to the late T. Pakula for fruitful collaboration. 


\section{References}

[1] D.A. Tomalia, H. Baker, J. R. Dewald, M. Hall, G. Kallos, S. Martin, J. Roeck, J. Ryder, P. Smith, Polym. J. 17, 117 (1985).

[2] E.W. Buhleier, W. Wehner, F. Vogtle, Synthesis 1978, 155 (1978).

[3] www.azonano.com/details.asp?ArticleID=1142\#_Dendrimers_(Organic_Nanoparticles).

[4] C.F. Welch, D.A. Hoagland, Langmuir 19, 1082 (2003).

[5] G. Nisato, R. Ivkov, E.J. Amis, Macromolecules 33, 4172 (2000).

[6] K.M.A. Rahman, C.J. Durning, N.J. Turro, D.A. Tomalia, Langmuir 16, 10154 (2000).

[7] X. Li, T. Imae, D. Leisner, M.A. López-Quintela, J. Phys. Chem. B 106, 12170 (2002).

[8] H. Zhang, P.L. Dubin, J. Ray, G.S. Manning, C.N. Moorefield, G.R. Newkome, J. Phys. Chem. B 103, 2347 (1999).

[9] D. Leisner, T. Imae, J. Phys. Chem. B 108, 1798 (2004).

[10] E.G. Timoshenko, Y.A. Kuznetsov, R. Connolly, J. Chem. Phys. 117, 9050 (2002).

[11] E.N. Govorun, K.B. Zeldovich, A.R. Khokhlov, Macromol. Theory Simul. 12, 705 (2003).

[12] P. Welch, M. Muthukumar, Macromolecules 31, 5892 (1998).

[13] P. Welch, M. Muthukumar, Macromolecules 33, 6159 (2000).

[14] S.V. Lyulin, A.A. Darinskii, A.V. Lyulin, M.A. Michels, Macromolecules 37, 4676 (2004).

[15] B. Dünweg, M. Stevens, K. Kremer, in: Monte Carlo and Molecular Dynamics Simulations in Polymer Science, Ed. K. Binder, Oxford University Press, New York 1999, p. 125.

[16] H. Schiessel, P. Pincus, Macromolecules 31, 7953 (1998).

[17] G.S. Manning, J. Chem. Phys. 51, 924 (1969).

[18] G.S. Manning, J. Chem. Phys. 51, 934 (1969).

[19] G.S. Manning, J. Chem. Phys. 51, 3249 (1969).

[20] G.S. Manning, J. Chem. Phys. 89, 3773 (1988).

[21] M.J. Stevens, K. Kremer, J. Chem. Phys. 103, 1669 (1995).

[22] J. Takashima, M. Takasu, Y. Hiwatari, Phys. Rev. A 40, 2706 (1989).

[23] R.G. Winkler, M. Gold, P. Reineker, Phys. Rev. Lett. 80, 3731 (1998).

[24] N.V. Brilliantov, D.V. Kuznetsov, R. Klein, Phys. Rev. Lett. 81, 1433 (1998).

[25] J.M. Victor, J.P. Hansen, Europhys. Lett. 3, 1161 (1987).

[26] J.P. Donley, J. Chem. Phys. 116, 5315 (2002).

[27] M. Deserno, C. Holm, S. May, Macromolecules 33, 199 (2000).

[28] A.V. Dobrynin, M. Rubinstein, Macromolecules 34, 1964 (2001).

[29] H.J. Limbach, C. Holm, J. Chem. Phys. 114, 9674 (2001). 
[30] M.J. Stevens, S. Plimpton, Eur. Phys. J. B 2, 341 (1998).

[31] R. Chang, A. Yethiraj, J. Chem. Phys. 118, 6634 (2003).

[32] T. Hofmann, R.G. Winkler, P. Reineker, J. Chem. Phys. 114, 10181 (2001).

[33] M. Muthukumar, J. Chem. Phys. 120, 9343 (2004).

[34] S. Liu, M. Muthukumar, J. Chem. Phys. 116, 9975 (2002).

[35] M. Majtyka, J. Kłos, J. Phys., Condens. Matter 18, 3581 (2006).

[36] A.A. Gurtovenko, S.V. Lyulin, M. Karttunen, L. Vattulainen, J. Chem. Phys. 124, 094904 (2006) and references therein.

[37] M.P. Allen, D.J. Tildesley, Computer Simulation of Liquids, Clarendon Press, Oxford 1984.

[38] N. Meropolis, A.W. Rosenbluth, N.N. Rosenbluth, A.H. Teller, E. Teller, J. Chem. Phys. 21, 1087 (1953).

[39] R.L. Lescanec, M. Muthukumar, Macromolecules 24, 4892 (1991).

[40] S. Rathgeber, T. Pakula, V. Urban, J. Chem. Phys. 121, 3840 (2004).

[41] D. Boris, M. Rubinstein, Macromolecules 29, 7251 (1996).

[42] V. Lyulin, G.R. Davies, D.B. Adolf, Macromolecules 33, 6899 (2000).

[43] K. Karatasos, D.B. Adolf, G.R. Davies, J. Chem. Phys. 115, 5310 (2001).

[44] M.L. Mansfield, L.I. Klushin, Macromolecules 26, 4262 (1993).

[45] M.F. Ottaviani, F. Montalti, N.J. Turro, D.A. Tomalia, J. Phys. Chem. B 101, 158 (1997).

[46] F. Gröhn, B.J. Bauer, Y.A. Akpalu, C.L. Jackson, E.J. Amis, Macromolecules 33, 6042 (2000).

[47] F. Gröhn, G. Kim, B.J. Bauer, E.J. Amis, Macromolecules 34, 2179 (2001).

[48] K. Vassilev, W.T. Ford, J. Polym. Sci. A 37, 2727 (1999).

[49] K. Esumi, A. Suzuki, N. Aihara, K. Usui, K. Torigoe, Langmuir 14, 3157 (1998). 